\title{
Microencapsulation extends mycelial viability of Streptomyces lividans 66 and increases enzyme production
}

\author{
Boris Zacchetti ${ }^{\dagger}$, Agathoklis Andrianos ${ }^{\dagger}$, Dino van Dissel ${ }^{\dagger}$, Evelien de Ruiter, Gilles P. van Wezel \\ and Dennis Claessen ${ }^{*}$ (D)
}

\begin{abstract}
Background: Filamentous bacteria of the genus Streptomyces produce a large arsenal of industrially relevant antibiotics and enzymes. The industrial production of these molecules occurs in large fermenters, where many streptomycetes form dense mycelial networks called pellets. Pellets are characterized by slow growth and inefficient nutrient transfer and therefore regarded as undesirable from the perspective of productivity. Although non-pelleting strains have increased growth rates, their morphology also leads to a dramatic increase in the viscosity of the culture broth, which negatively impacts the process dynamics.

Results: Here, we applied immobilization of Streptomyces lividans 66 using alginate as semi-solid matrix. This alginatemediated micro-encapsulation increased the production of the extracellular enzyme tyrosinase more than three-fold. The increased production was accompanied by extended viability of the mycelium and a dramatic reduction in the release of intracellular proteins into the culture broth.
\end{abstract}

Conclusions: Our data demonstrate the utility of micro-encapsulation as a powerful technique to achieve higher yields and lower downstream-processing costs of streptomycetes.

Keywords: Streptomyces, Microencapsulation, Enzyme production

\section{Background}

Filamentous organisms are widely used in the field of industrial biotechnology. Of particular relevance are the streptomycetes, multicellular bacteria that produce a vast array of useful metabolites, including over half of the clinically relevant antibiotics, various antitumor agents, immunosuppressants, anthelminthics, antifungals, herbicides and insecticides [1, 2]. In addition, streptomycetes produce and secrete a wealth of extracellular hydrolases, which they employ to degrade the big majority of natural occurring polymers [3]. Streptomycetes grow as filaments (hyphae) that occasionally branch and establish extended cellular networks called mycelia. Growth under industrial settings is characterized by the formation of dense mycelial

\footnotetext{
* Correspondence: d.claessen@biology.leidenuniv.nl

${ }^{\dagger}$ Equal contributors

Microbial Biotechnology, Institute of Biology Leiden, Leiden University, Sylviusweg 72, 2333, BE, Leiden, The Netherlands
}

networks called pellets $[4,5]$, a phenomenon posing significant drawbacks in terms of industrial applicability. More specifically, pellets only actively grow at the periphery, which limits productivity [6, 7]. Recent work has indicated that it is possible to circumvent pellet formation in Streptomyces lividans by interfering with the biosynthesis of extracellular glycans produced by the cslA-glxA and matAB loci $[6,8]$. These glycans mediate the adherence of hyphae, hence leading to the formation of dense clumps and pellets [4]. Deletion mutants of these genes do not form pellets and grow in a dispersed manner. This increases growth and enzyme production rates [6], but comes with the offset of a higher viscosity of the culture broth (our unpublished data). To further complicate matters, pelleted growth appears to be essential at least for the production of some antibiotics [911]. All in all, the mycelial mode-of-growth of streptomycetes results in production processes that are 
characterized by a complex rheology. This translates into suboptimal mass-transfer properties, heat transfer problems, mechanical and oxidative stress $[5,10,12]$.

An attractive alternative to classical fermentations is offered by micro-encapsulation, via the immobilization of cells in a semi-solid scaffold, often sodium alginate [13]. The behavior of a number of micro-organisms has been characterized in this immobilized state, which was found to bear several advantages. In comparison to freeliving cells, immobilized cells are better protected from harsh environmental conditions [14-17] and enhanced productivity has been reported [18, 19]. Additionally, immobilized cells are readily recycled or filtered, which reduces the yield loss associated with the accumulation of biomass and facilitates downstream processing [20]. In this study, we report that micro-encapsulation of the industrial workhorse Streptomyces lividans enhances heterologous production and purity of the extracellular enzyme tyrosinase. Our data indicate that microencapsulation provides protection against shear stress, thereby maintaining mycelial viability and integrity. This in turn stimulates production and reduces contaminations with proteins released by cell lysis.

\section{Results}

Growth of streptomycetes in calcium-alginate microcapsules extends the viability of the mycelium

To study the effect of microencapsulation on the growth of streptomycetes, spores of S. lividans, S. coelicolor, S. venezuelae and $S$. griseus were immobilized in alginate microcapsules (see Materials and Methods). Following the immobilization step, the encapsulated spores were

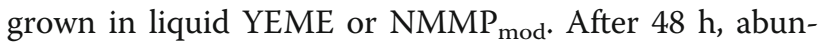
dant mycelial growth was detected for all strains in both media (Fig. 1 and Additional file 1: Figure S1). In YEME medium, the encapsulated mycelium of all strains formed highly compact mycelial clumps, while portions of the mycelium that had started to grow out of the microcapsules adopted a more relaxed morphology, whereby individual hyphae could be discerned (Additional file 1: Figure S1). In $\mathrm{NMMP}_{\text {mod. }}$ medium, the encapsulated mycelium formed less compact clumps (Fig. 1). The mycelium of all strains started to grow outside of the microcapsules after $48 \mathrm{~h}$ of growth, and became visible as 'spikes' protruding from their edges. With the exception of S. coelicolor, non-encapsulated mycelium was found in all strains in the liquid during the late stages of growth (Fig. 1 and Additional file 2: Figure S2). This phenomenon was particularly evident in S. griseus and S. venezuelae at $48 \mathrm{~h}$ of growth, while it became apparent in S. lividans at $96 \mathrm{~h}$ of growth (Additional file 2: Figure S2).

The less compact morphology of the encapsulated mycelium in $\mathrm{NMMP}_{\text {mod }}$ compared to YEME medium, which we anticipated to be beneficial for enzyme production, was a reason to further only use $\mathrm{NMMP}_{\text {mod }}$ medium. We also focused on S. lividans, given the prominent role of this strain for the industrial production of enzymes. To analyze how encapsulation affects mycelial viability, we performed a LIVE/DEAD analysis on the encapsulated and free-living mycelium (Fig. 2). Interestingly, a major fraction of the mycelium present in, or associated with the microcapsules after 48 and $72 \mathrm{~h}$ of growth was viable, as derived from the pronounced staining with Syto-9. In contrast, the presence of damaged mycelium, indicated by the red PI stain, occurred significantly earlier in the free-growing mycelium, either cultivated in the presence or absence of a metal coil (used to counteract aggregation and induce fragmentation by shear). When free-growing mycelium of S. lividans was cultivated in the absence of a metal coil in the culture flask, we noticed that the

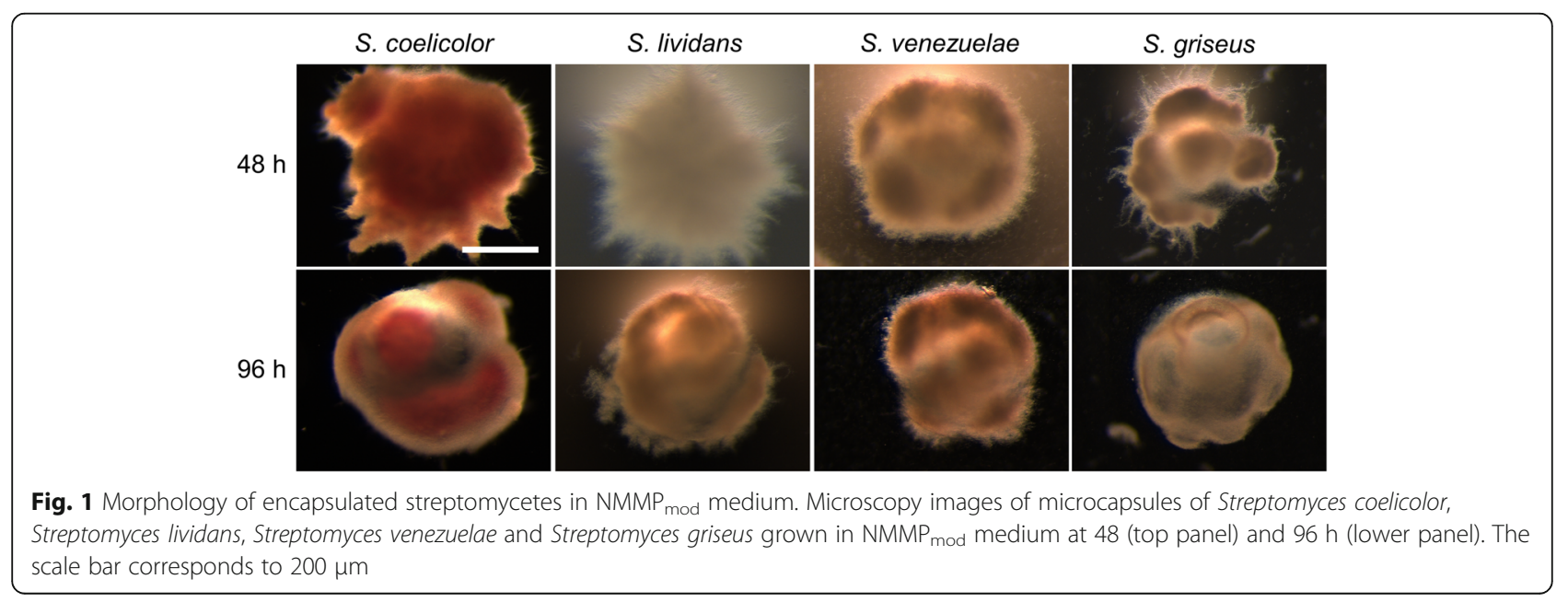




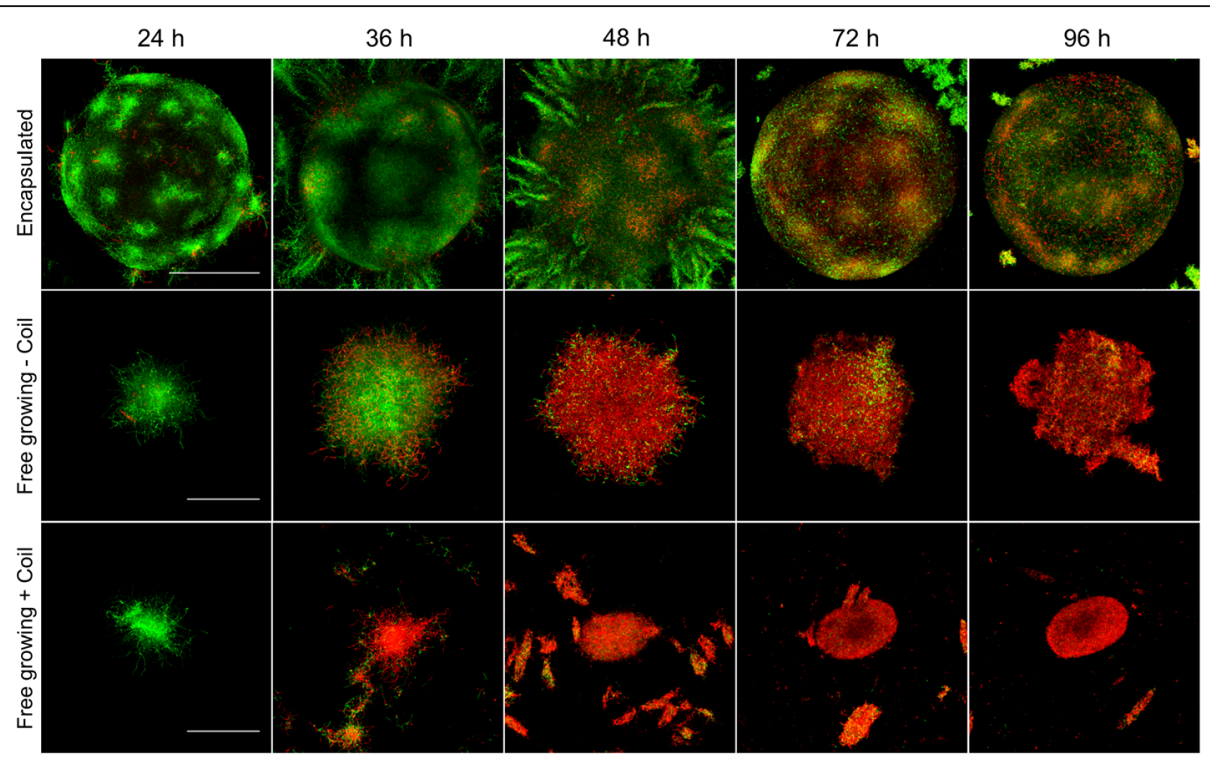

Fig. 2 Microencapsulation reduces mycelial damage. LIVE/DEAD staining of S. lividans grown in microcapsules (top row), or non-encapsulated in the absence (middle row) or presence (bottom row) of a metal coil. Mycelium stained with Syto9 (green) represents viable mycelium, while propidium iodide-stained mycelium (red) is damaged. Whereas abundant viable mycelium is visible in the encapsulated state at $48 \mathrm{~h}$ of growth, the non-encapsulated mycelium appears highly damaged. Note that the mycelium grown in the presence of the coil appears already damaged after $36 \mathrm{~h}$. The scale bar represents $200 \mu \mathrm{m}$ (top panel) or $100 \mu \mathrm{m}$ (middle and bottom panel)

occurrence of damaged mycelium was evident after $48 \mathrm{~h}$ of growth, and apparently delayed in comparison to the mycelium suffering from coil-induced shear.

\section{Microencapsulation increases the heterologous production of tyrosinase}

To test the effect of microencapsulation on heterologous enzyme production, we introduced plasmid pIJ703 into S. lividans 66 [21]. This plasmid contains the melC2 gene of Streptomyces antibioticus, encoding an extracellular tyrosinase that is secreted via the twin arginine translocation pathway [22]. Transformants were selected based on their ability to form the pigmented compound melanin; one of these, hereinafter called S. lividans pIJ703, was selected for further analysis. S. lividans pIJ703 was encapsulated, after which the tyrosinase production was assayed and compared to the non-encapsulated controls (Fig. 3). Significantly enhanced activity ( $P$-value < 0.0001 ) was detected in the supernatant between 40 and $54 \mathrm{~h}$ of growth when S. lividans pIJ703 was grown in microcapsules, with a more than three-fold increase in comparison to the non-encapsulated strain. The highest tyrosinase activity in the supernatants of the encapsulated strain peaked after $48 \mathrm{~h}$ of growth, followed by a slow and gradual decrease. However, tyrosinase activity was still detectable at $72 \mathrm{~h}$ of growth. In the non-encapsulated state, the tyrosinase activity peaked at approximately $34 \mathrm{~h}$ of growth, after which a rapid decline was detected.
After $50 \mathrm{~h}$, tyrosinase activity was barely detectable. Given that the growth rate of the encapsulated mycelium could not be assessed, we measured glucose consumption over time (Additional file 3: Figure S3). The consumption of glucose did not show significant differences between the three culture types, suggesting that the mycelia grew at comparable rates.

We also qualitatively analyzed the extracellular proteins present in the culture supernatants (Fig. 4a). An SDS-PAGE analysis indicated that the supernatant of the

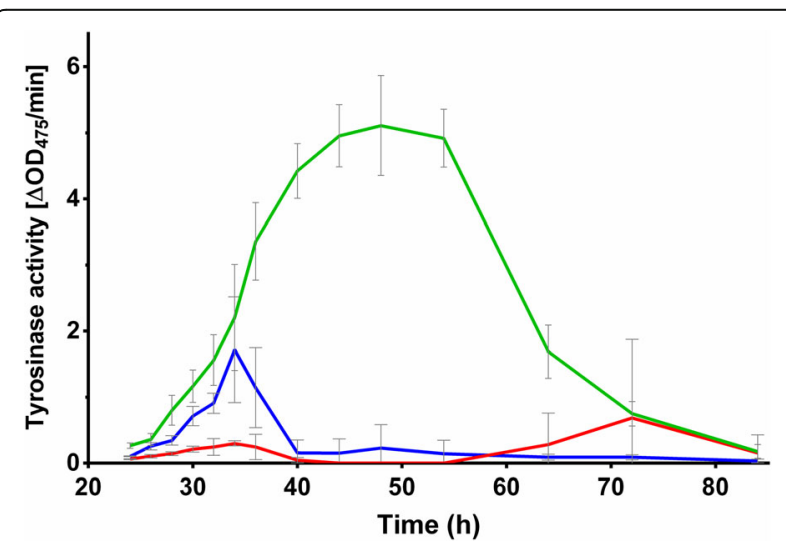

Fig. 3 Microencapsulation increases tyrosinase activity in the supernatant. Lines represent the tyrosinase activity present in the supernatant of S. lividans plJ703 grown encapsulated (green), or non-encapsulated in the absence (red) or presence (blue) of a coil. The highest activity was observed in the culture broth of the encapsulated mycelium after $48 \mathrm{~h}$ of growth 

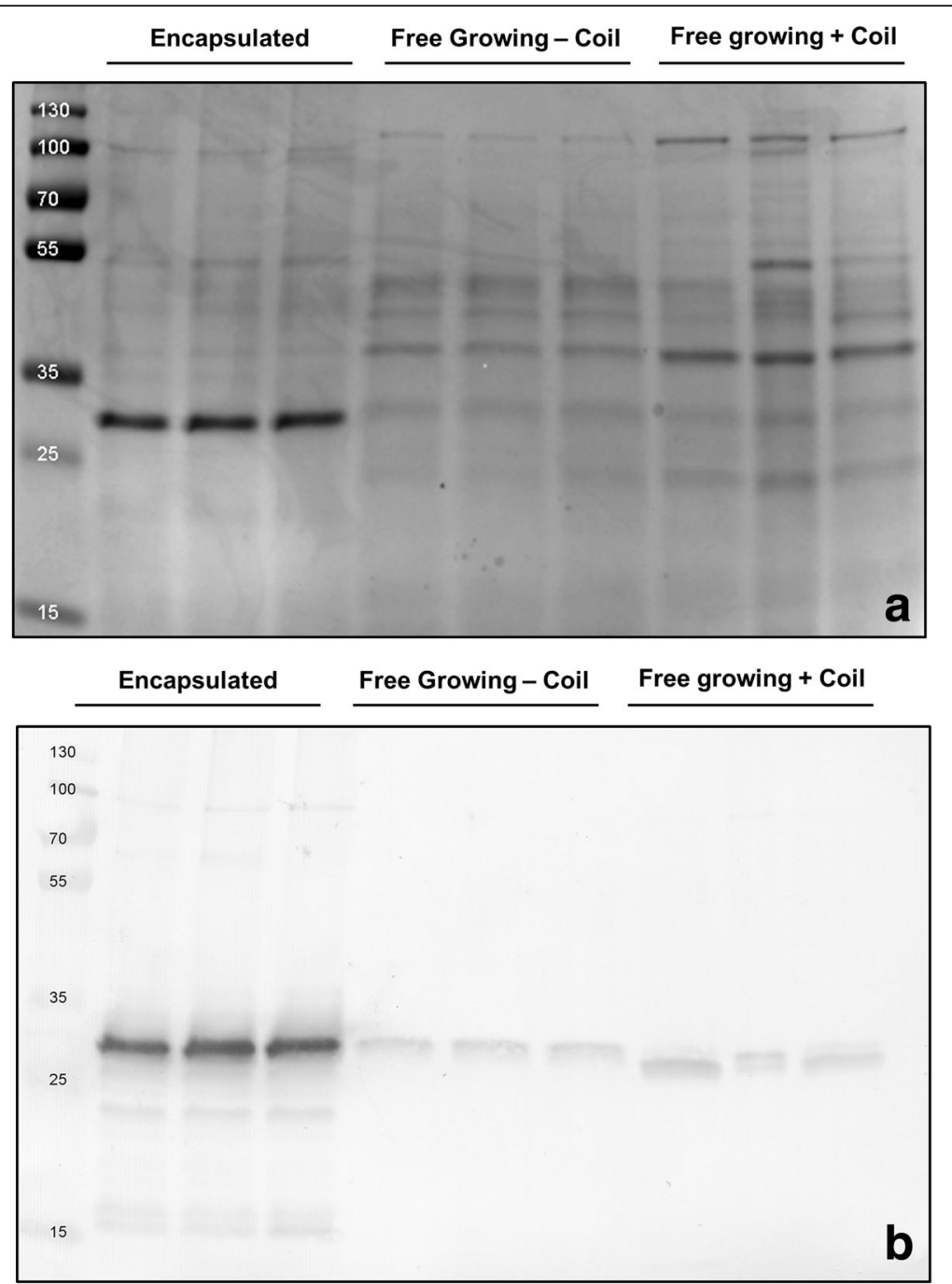

Fig. 4 Microencapsulation increases tyrosinase purity in the culture broth. a SDS-page showing the protein profiles in the supernatants of cultures of S. lividans plJ703, grown encapsulated (lanes 2-4), or non-encapsulated in the absence (lanes 5-7) and presence (lanes 8-10) of a metal coil. All cultures were performed in triplicate. Molecular weight markers (lane 1) are indicated in $\mathrm{kDa}$. b Western analysis of the abovementioned supernatants using an anti-Tyrosinase antibody. Molecular weight markers are indicated in $\mathrm{kDa}$

encapsulated strain contained an abundant protein with an apparent molecular weight equal to that of tyrosinase $(\sim 30 \mathrm{kDa})$ after $48 \mathrm{~h}$ of growth, corresponding to the time point where most tyrosinase activity was detected. Conversely, the protein profiles in the supernatants of the non-encapsulated control cultures were more complex and showed a large number of proteins (Fig. 4a). Western analysis was used to verify that the dominant protein in the supernatant of the encapsulated strain was in fact tyrosinase (Fig. 4b). Consistent with the measured activities in the supernatant, only small amounts of tyrosinase were detected in the non-encapsulated strains. Taken together, these data demonstrate that encapsulation enhances the production of heterologously expressed tyrosinase.

\section{Discussion}

Streptomycetes are proficient producers of enzymes and antibiotics. For industrial production processes, these organisms are usually grown as liquid-grown cultures in large scale fermenters. The growth of streptomycetes under these conditions is marked by the formation of mycelial particles that consist of interconnected hyphae $[4,23]$. Industrial fermenters are typically stirred at high speeds to provide homogeneous mixing but also to ensure that sufficient oxygen and nutrients are available to the growing biomass. This vigorous mixing comes at the cost of severe shear stress, which can cause fragmentation and lysis of the mycelium [24-26]. The concomitant release of intracellular contents into the culture broth thereby complicates product purification [27]. 
We here show that microencapsulation is a valuable and alternative approach circumventing some of the negative aspects of classical fermentations, consistent with earlier observations [19]. Microencapsulation physically separates a large fraction of the mycelium from the liquid environment, with the exception of the small mycelial fragments that grow out of the capsules at late time points. While the calciumalginate is permeable to small molecules [28], the encapsulated mycelium is protected from extrinsic mechanical stress. Our experiments demonstrate that the viability of the mycelium is prolonged inside the microcapsules, which is in agreement with earlier observations in S. coelicolor [19]. We conclude that this effect is obtained by reducing the degree of shear stress encountered by the mycelium. The earlier occurrence of dead mycelium in non-encapsulated cultures performed with metal coils as compared to those without is also indicative of this fact. Besides extending viability, microencapsulation dramatically increased the production and purity of heterologously produced tyrosinase. The higher production was not only evident from the amount of this protein in the supernatant, but also from measurement of its specific activity throughout growth. More specifically, the amount of active tyrosinase was more than threefold increased when the mycelium was grown inside the microcapsules.

Shear-induced cell lysis can be a major cause for the dissipation of substrate in streptomycetes [25]. This, accompanied by the observation that the trend in carbon consumption was similar under all conditions, suggests that the encapsulated mycelium invests more energy in production rather than in other processes, such as those related to cell repair and maintenance. The higher purity of the extracellular tyrosinase not only supports that micro-encapsulation reduces cell lysis, but also poses another major benefit: the decreased number of contaminants facilitates product purification and increases the efficiency of the production process.

\section{Conclusions}

Our work serves as proof-of-concept showing that the microencapsulation of streptomycetes prolongs mycelial viability and enhances the production and purity of enzymes. One issue to overcome is the need to scale up to allow larger scale production with encapsulated strains. Considering this, we anticipate that our approach might be particularly suitable for the production of high-value natural products and enzymes by streptomycetes and possibly other filamentous organisms.

\section{Methods}

\section{Strains and culture conditions}

Streptomyces coelicolor A3(2) M145 [29], Streptomyces

lividans 66 [21] and Streptomyces venezuelae diversa were obtained from the John Innes Centre strain collection, and Streptomyces griseus DSM40236 from the Deutsche Sammlung von Mikroorganismen und Zellkulturen (DSMZ). MS agar plates [30] were used to prepare spore suspensions of Streptomyces strains and to determine colony forming units (CFU) for the spore stocks. For liquid-grown cultures, YEME medium [30] or a modified NMMP medium $\left(\mathrm{NMMP}_{\text {mod }}\right)$ were used. The buffer system of $\mathrm{NMMP}_{\text {mod }}$ was optimized to avoid the detrimental effect of phosphates on the integrity of the alginate microcapsules [31]. For the preparation of 11 $\mathrm{NMMP}_{\text {mod }}$ medium, $100 \mathrm{ml} 0.25 \mathrm{M}$ TES buffer ( $\mathrm{pH}$ 7.2), $10 \mathrm{ml} 0.1 \mathrm{M} \mathrm{Na}-\mathrm{K}$ buffer (pH 6.8), $25 \mathrm{ml} \mathrm{20 \%}$ glucose and $65 \mathrm{ml}$ milliQ water were added to $800 \mathrm{ml}$ NMMP basis [30]. For experiments using strains containing the tyrosinase-expressing plasmid pIJ703 [22], $10 \mu \mathrm{M} \mathrm{CuSO}_{4}$ were added to the growth medium. All cultures were grown in a total volume of $100 \mathrm{ml}$ of liquid medium contained in $250 \mathrm{ml}$ Erlenmeyer flasks. Cultures were grown in an orbital shaker set at $30{ }^{\circ} \mathrm{C}$ and $160 \mathrm{rpm}$. Unless differently stated, all experiments were performed in duplicate. For microencapsulation experiments, on average 75 viable spores were incorporated into every capsule with an average diameter of $415 \mu \mathrm{m}$. To this end, spores were suspended in sterile liquid sodium alginate and thoroughly mixed before the preparation of microcapsules. A total of $5 \mathrm{ml}$ of alginate microcapsules was used to inoculate $100 \mathrm{ml}$ of medium (see below). We calculated the equivalent number of spores, which we used to inoculate all other cultures, and which corresponded to $10^{5}$ spores per $\mathrm{ml}$ of medium.

\section{Microscopy}

A Leica MZ12 stereo microscope was used for the visualization of microcapsules and encapsulated mycelium. For the visualization of live and dead mycelium, samples were stained with Syto-9 and propidium iodide (PI) (Invitrogen). To this end, pellets and microcapsules were briefly sedimented via centrifugation $(10 \mathrm{~min}$ at $2000 \mathrm{rpm}$ at room temperature) and resuspended in PBS, to which Syto-9 and PI were added to a final concentration of $5 \mu \mathrm{M}$ and $15 \mu \mathrm{M}$ respectively. After mixing and incubating for $10 \mathrm{~min}$ in the dark at $30{ }^{\circ} \mathrm{C}$, samples were analyzed using a Zeiss LSM 5 EXCITER confocal microscope. Stained samples were excited at 488 and $543 \mathrm{~nm}$ for Syto-9 and PI, respectively. The fluorescence emission of Syto-9 was monitored in the region between 505 and $545 \mathrm{~nm}$, while a long-pass filter of $560 \mathrm{~nm}$ was used to detect PI [32]. The pictures shown in Fig. 2 represent Z-stacks of at least 15 layers in 
the specimen with a slice thickness of $7 \mu \mathrm{m}$ for microcapsules and $4 \mu \mathrm{m}$ for pellets.

\section{Encapsulation of Streptomyces spores in calcium alginate}

Sodium alginate (Sigma-Aldrich, CAS:9005-38-3) was dissolved under constant stirring for $1 \mathrm{~h}$ in milliQ water to obtain a $2 \%$ solution. To remove undissolved microparticles and other contaminants, the obtained solution was passed through two different filters. The first filter had a pore size of $1.2 \mu \mathrm{m}$ (GE Healthcare, CatNo:1822047) and was used in a vacuum filtration apparatus (PALL Magnetic Filter Funnel). The filtrate was then filter-sterilized using a syringe filter with a pore size of $0.22 \mu \mathrm{m}$ (Sarstedt). For the production of calciumalginate microcapsules, a home-made device was used similar to that described in [33]. A schematic representation is shown in Additional file 4: Figure S4. This apparatus is based on a coaxial gas-flow extrusion principle, with sterile air as the used gas. The air flow was regulated via a controller (Kytola, Model E) and was set at 31 per minute, thus yielding alginate particles with an average diameter of $415 \mu \mathrm{m}( \pm 12.3 \mu \mathrm{m}$; based on analyzing 150 particles). A constant alginate flow was obtained by using a syringe pump (Fisher-Scientific) set at $30 \mathrm{ml} / \mathrm{h}$. The microcapsules were produced by dispersing the extruded alginate using a home-made nozzle that allowed co-axial laminar flow. While the alginate constitutes the inner sheath, air is flown in a co-axial fashion and determines the rate of formation of the alginate drops. This allowed control over the volume of the falling droplets, which were collected into a gently stirred $200 \mathrm{mM} \mathrm{CaCl}_{2}$ solution. The cross-links formed through sodium/calcium ion exchange almost instantly transformed the liquid drops into gel-like microcapsules. The alginate microcapsules were left to harden in the stirring $200 \mathrm{mM} \mathrm{CaCl}_{2}$ solution for $5 \mathrm{~min}$. The obtained suspension of calcium-alginate microcapsules was then filtered using a vacuum filtration apparatus (PALL, GE Healthcare filters as above), after which the microcapsules were washed three times with $500 \mathrm{ml}$ sterile demiwater.

\section{Glucose measurement assay}

Glucose concentrations were determined using a commercial kit (Megazyme, HK/G6P-DH), according to the instructions of the manufacturer.

\section{Tyrosinase activity assay}

The specific activity of tyrosinase produced by S. lividans harboring pIJ703 was determined by following the conversion of L-3,4-dihydroxyphenylalanine spectrophotometrically at a wavelength of $475 \mathrm{~nm}$, as described earlier [34]. Graphpad Prism 6.01 was used for performing a one-way analysis of variance (ANOVA) followed by a Bonferroni correction.

\section{SDS-PAGE and western blot analyses}

Supernatants of liquid-grown cultures were harvested after $48 \mathrm{~h}$ of growth. The culture samples were first centrifuged for $10 \mathrm{~min}$ at $5000 \mathrm{rpm}$ and $4{ }^{\circ} \mathrm{C}$, after which the supernatants were filtered through $0.22 \mu \mathrm{m}$ syringe filters (Sarstedt), to remove any possible contaminants (e.g whole cells, spores). Extracellular proteins were concentrated via acetone precipitation. Briefly, $1.2 \mathrm{ml}$ of cold acetone $\left(-20{ }^{\circ} \mathrm{C}\right)$ were added to $300 \mu \mathrm{l}$ of supernatant sample. Following thorough mixing, the sample was kept at $-20{ }^{\circ} \mathrm{C}$ for $1 \mathrm{~h}$ and subsequently centrifuged at $13,000 \mathrm{rpm}$ for $10 \mathrm{~min}$ at $0{ }^{\circ} \mathrm{C}$. Subsequently, the liquid was removed without disturbing the protein pellet, after which $500 \mu \mathrm{l}$ of cold acetone were added. After a second centrifugation step, the acetone was removed and the pellet was dried at $37{ }^{\circ} \mathrm{C}$ for $10 \mathrm{~min}$. The obtained protein pellets were dissolved in $30 \mu \mathrm{l}$ of $10 \mathrm{mM}$ Tris- $\mathrm{HCl}$ buffer ( $\mathrm{pH}$ 8.0). A Bradford analysis was used to determine the protein concentrations in the obtained samples, and $2 \mu \mathrm{g}$ of proteins were used for separation by SDS-PAGE on precast $12 \%$ miniprotean TGX Gels (BioRad) at $205 \mathrm{~V}, 200 \mathrm{~mA}$ for approximately $50 \mathrm{~min}$. Proteins were transferred to polyvinylidene difluoride (PVDF) membranes (GE Healthcare) and incubated overnight with antiTyrosinase polyclonal antibodies (1:25,000 dilution). Following $1 \mathrm{~h}$ of incubation with goat anti-rabbit alkaline phosphatase, detection was carried out with NBT/BCIP. The relative quantification of proteins on SDS-pages was performed using ImageJ (version $1.48 \mathrm{f})$.

\section{Additional files}

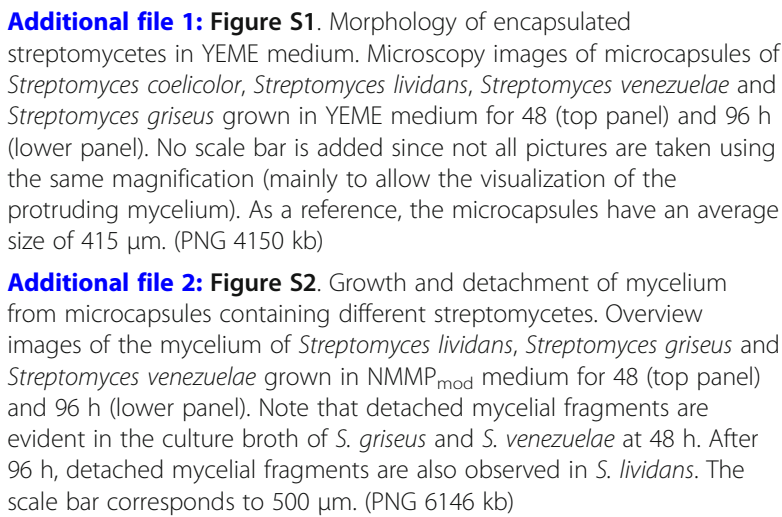

Additional file 2: Figure S2. Growth and detachment of mycelium from microcapsules containing different streptomycetes. Overview images of the mycelium of Streptomyces lividans, Streptomyces griseus and Streptomyces venezuelae grown in NMMP mod medium for 48 (top panel) and $96 \mathrm{~h}$ (lower panel). Note that detached mycelial fragments are evident in the culture broth of S. griseus and S. venezuelae at $48 \mathrm{~h}$. After $96 \mathrm{~h}$, detached mycelial fragments are also observed in S. lividans. The scale bar corresponds to $500 \mu \mathrm{m}$. (PNG $6146 \mathrm{~kb}$ )

Additional file: $\mathbf{3}$ Figure S3. Glucose consumption by encapsulated and non-encapsulated mycelium. The residual glucose concentrations (in $\mathrm{g} / \mathrm{L}$ ) in $\mathrm{NMMP}_{\text {mod }}$ medium are shown when Streptomyces lividans plJ703 
is grown in micro-capsules (green), or non-encapsulated in the absence (red) and presence (blue) of a metal coil. (PNG $179 \mathrm{~kb}$ )

Additional file 4: Figure S4. Schematic representation of the microencapsulation apparatus. A constant flow of dissolved alginate is maintained with a syringe pump set at $30 \mathrm{ml} \mathrm{h}^{-1}$, while the air flow is regulated with an air flow controller set at $3 \mathrm{~L} \mathrm{~h}^{-1}$. The sodium alginate droplets that detach form the nozzle fall in a gently-stirred solution of $200 \mathrm{mM} \mathrm{CaCl}$, leading to formation of the microcapsules via ion exchange. The nozzle functions according to the coaxial gas-flow extrusion principle [33]. (PNG $155 \mathrm{~kb})$

\section{Acknowledgements}

Not applicable.

\section{Funding}

This work was supported by grant 12957 from the Netherlands Technology Foundation to DC.

\section{Availability of data and materials}

Not applicable.

\section{Authors' contributions}

BZ performed the experiments with the help of AA, DVD and ER. DC and BZ conceived the study. BZ and DC wrote the manuscript with the help of GPvW. All authors read and approved the final manuscript.

\section{Ethics approval and consent to participate}

Not applicable.

\section{Consent for publication}

Not applicable.

\section{Competing interests}

The authors declare that they have no competing interests.

\section{Publisher's Note}

Springer Nature remains neutral with regard to jurisdictional claims in published maps and institutional affiliations.

Received: 17 October 2017 Accepted: 7 March 2018

Published online: 12 March 2018

\section{References}

1. Barka EA, Vatsa P, Sanchez L, Gavaut-Vaillant N, Jacquard C, MeierKolthoff J, Klenk HP, Clément C, Oudouch Y, van Wezel GP. Taxonomy, physiology, and natural products of the Actinobacteria. Microbiol Mol Biol Rev. 2016;80(1):1-43.

2. Bérdy J. Bioactive microbial metabolites. J Antibiot (Tokyo). 2005;58(1):1-26.

3. Vrancken $K$, Anné J. Secretory production of recombinant proteins by Streptomyces. Future Microbiol. 2009;4(2):181-8.

4. Zacchetti B, Willemse J, Recter B, van Dissel D, van Wezel GP, Wösten HAB, Claessen D. Aggregation of germlings is a major contributing factor towards mycelial heterogeneity of Streptomyces. Sci Rep. 2016;6:27045.

5. Celler K, Picioreanu C, van Loosdrecht MCM, van Wezel GP. Structured morphological modeling as a framework for rational strain design of Streptomyces species. Antonie Van Leeuwenhoek. 2012;102(3):409-23.

6. van Dissel D, Claessen D, Roth M, van Wezel GP. A novel locus for mycelial aggregation forms a gateway to improved Streptomyces cell factories. Microb Cell Factories. 2015;14(1):44.

7. Liu L, Yang H, Shin HD, Li J, Du G, Chen J. Recent advances in recombinant protein expression by Corynebacterium, Brevibacterium, and Streptomyces: from transcription and translation regulation to secretion pathway selection. Appl Microbiol Biotechnol. 2013;97(22):9597-608.

8. Chaplin AK, Petrus MLC, Mangiameli G, Hough MA, Svistunenko DA, Nicholls P, Claessen D, Vijgenboom E, Worrall JAR. GlXA is a new structural member of the radical copper oxidase family and is required for glycan deposition at hyphal tips and morphogenesis of Streptomyces lividans. Biochem J. 2015;469(3):433-44.
9. Wardell JN, Stocks SM, Thomas CR, Bushell ME. Decreasing the hyphal branching rate of Saccharopolyspora erythraea NRRL 2338 leads to increased resistance to breakage and increased antibiotic production. Biotechnol Bioeng. 2002;78(2):141-6.

10. van Dissel D, Claessen D, van Wezel GP. Morphogenesis of Streptomyces in submerged cultures. Adv Appl Microbiol. 2014;89:1-45.

11. Pickup KM, Bushell ME. Non-fragmenting variants of Streptomyces hyphae have enhanced activity of an enzyme (phospho- $\mathrm{N}$-acetylmuramyl pentapeptide translocase) in peptidoglycan biosynthesis. J Ferment Bioeng. 1995;79(3):247-51.

12. Nielsen J. Modelling the morphology of filamentous microorganisms. Trends Biotechnol. 1996;14(11):438-43.

13. Park JK, Chang HN. Microencapsulation of microbial cells. Biotechnol Adv. 2000;18(4):303-19.

14. Buzas Z, Dallmann K, Szajani B. Influenc of pH on the growth and ethanol production of free and immobilized Saccharomyces cerevisiae cells. Biotechnol Bioeng. 1989;34(6):882-4.

15. Russo A, Basaglia M, Tola E, Casella S. Survival, root colonisation and biocontrol capacities of Pseudomonas fluorescens F113 LacZY in dry alginate microbeads. J Ind Microbiol Biotechnol. 2001;27(6):337-42.

16. McCabe BK, Kuek C, Gordon GL, Phillips MW. Production of beta-glucosidase using immobilised Piromyces sp. KSX1 and Orpinomyces sp. 478P1 in repeatbatch culture. J Ind Microbiol Biotechnol. 2003;30(4):205-9.

17. Briceno G, Fuentes MS, Rubilar O, Jorquera M, Tortella G, Palma G, Amoroso MJ, Diez MC. Removal of the insecticide diazinon from liquid media by free and immobilized Streptomyces sp. isolated from agricultural soil. J Basic Microbiol. 2015:55(3):293-302.

18. Anisha GS, Prema P. Cell immobilization technique for the enhanced production of a-galactosidase by Streptomyces griseoloalbus. Bioresour Technol. 2008;99(9):3325-30.

19. López-García MT, Rioseras B, Yagüe P, Álvarez JR, Manteca Á. Cell immobilization of Streptomyces coelicolor: effect on differentiation and actinorhodin production. Int Microbiol. 2014;17(2):75-80.

20. Najafpour G, Younesi H, Syahidah Ku Ismail K. Ethanol fermentation in an immobilized cell reactor using Saccharomyces cerevisiae. Bioresour Technol. 2004:92(3):251-60.

21. Cruz-Morales P, Vijgenboom E, Iruegas-Bocardo F, Girard G, Yanez-Guerra LA, Ramos-Aboites HE, Pernodet JL, Anne J, van Wezel GP, Barona-Gomez F. The genome sequence of Streptomyces lividans 66 reveals a novel tRNAdependent peptide biosynthetic system within a metal-related genomic island. Genome Biol Evol. 2013;5(6):1165-75.

22. Katz E, Thompson CJ, Hopwood DA. Cloning and expression of the tyrosinase gene from Streptomyces antibioticus in Streptomyces lividans. J Gen Microbiol. 1983;129(9):2703-14.

23. van Veluw GJ, Petrus MLC, Gubbens J, de Graaf R, de Jong IP, van Wezel GP, Wösten HAB, Claessen D. Analysis of two distinct mycelial populations in liquid-grown Streptomyces cultures using a flow cytometry-based proteomics approach. Appl Microbiol Biotechnol. 2012;96(5):1301-12.

24. Large KP, Ison AP, Williams DJ. The effect of agitation rate on lipid utilisation and clavulanic acid production in Streptomyces clavuligerus. J Biotechnol. 1998:63(2):111-9.

25. Roubos JA, Krabben P, Luiten RGM, Verbruggen HB, Heijnen JJ. A quantitative approach to characterizing cell lysis caused by mechanical agitation of Streptomyces clavuligerus. Biotechnol Prog. 2001;17(2):336-47.

26. Mehmood N, Olmos E, Marchal P, Goergen JL, Delaunay S. Relation between pristinamycins production by Streptomyces pristinaespiralis, power dissipation and volumetric gas-liquid mass transfer coefficient, kLa. Process Biochem. 2010;45(11):1779-86.

27. Ohta N, Park YS, Yahiro K, Okabe M. Comparison of neomycin production from Streptomyces fradiae cultivation using soybean oil as the sole carbon source in an air-lift bioreactor and a stirred-tank reactor. J Ferment Bioeng. 1995:79(5):443-8.

28. Gaserod O, Sannes A, Skjak-Braek G. Microcapsules of alginate-chitosan. II. A study of capsule stability and permeability. Biomaterials. 1999;20(8): 773-83.

29. Bentley SD, Chater KF, Cerdeno-Tarraga AM, Challis GL, Thomson NR, James KD, Harris DE, Quail MA, Kieser H, Harper D, et al. Complete genome sequence of the model actinomycete Streptomyces coelicolor A3(2). Nature. 2002:417(6885):141-7.

30. Kieser T, Bibb MJ, Buttner MJ, Chater KF, Hopwood DA. Practical Streptomyces genetics. The John Innes Foundation: Norwich; 2000. 
31. Bhujbal SV, Paredes-Juarez GA, Niclou SP, de Vos P. Factors influencing the mechanical stability of alginate beads applicable for immunoisolation of mammalian cells. J Mech Behav Biomed Mater. 2014;37:196-208.

32. Willemse J, van Wezel GP. Imaging of Streptomyces coelicolor A3(2) with reduced autofluorescence reveals a novel stage of FtsZ localization. PLoS One. 2009:4(1):e4242.

33. Kontturi LS, Yliperttula M, Toivanen P, Maatta A, Maatta AM, Urtti A. A laboratory-scale device for the straightforward production of uniform, small sized cell microcapsules with long-term cell viability. J Control Release. 2011;152(3):376-81.

34. Lerch K, Ettinger L. Purification and characterization of a tyrosinase from Streptomyces glaucescens. Eur J Biochem. 1972;31(3):427-37.

Submit your next manuscript to BioMed Central and we will help you at every step:

- We accept pre-submission inquiries

- Our selector tool helps you to find the most relevant journal

- We provide round the clock customer support

- Convenient online submission

- Thorough peer review

- Inclusion in PubMed and all major indexing services

- Maximum visibility for your research

Submit your manuscript at www.biomedcentral.com/submit 